\title{
THE LANGLANDS QUOTIENT THEOREM FOR FINITE CENTRAL EXTENSIONS OF $p$-ADIC GROUPS
}

\author{
DubraVka BAN AND CHRIS JANTZEN \\ Southern Illinois University, USA and East Carolina University, USA
}

\begin{abstract}
In this paper, we prove the Langlands quotient theorem in the context of finite central extensions of connected, reductive $p$-adic groups.
\end{abstract}

\section{INTRODUCTION}

In this paper, we prove the Langlands quotient theorem in the context of finite central extensions of reductive $p$-adic groups (which includes the double cover $S \widetilde{p(2 n, F)}$ and the metaplectic covers of $G L(n, F)$, see $[15,18])$.

Suppose $G$ is the $F$-points of a connected, reductive group defined over a nonarchimedean local field $F$. The Langlands classification (Langlands quotient theorem) gives a bijective correspondence

$$
\operatorname{Irr}(G) \longleftrightarrow \operatorname{Lang}(G)
$$

between irreducible, admissible representations of $G$ and triples of Langlands data $([6,17,22])$. The Langlands classification was originally done in the context of connected real groups $([20])$. The proof for real groups given in [6] covers any real $G$ in Harish-Chandra's class, so applies to metaplectic covers. In this paper, we prove the Langlands classification for metaplectic covers of $p$-adic groups.

More precisely, let $G$ be the group of $F$-points of a connected, reductive group defined over a nonarchimedean local field $F$ of characteristic zero. Let

2010 Mathematics Subject Classification. 22E50, 11F70.

Key words and phrases. Metaplectic groups, Langlands quotient theorem, p-adic groups.

D.B. supported in part by NSF grant DMS-0601005 and by a Research Fellowship of the Alexander von Humboldt Foundation; C.J. supported in part by NSA grant H9823010-1-0162. 
$(\tilde{G}, \rho)$ be a finite central extension of $G$ as defined in section 2. In particular, we have a short exact sequence

$$
1 \longrightarrow C \longrightarrow \tilde{G} \stackrel{\rho}{\longrightarrow} G \longrightarrow 1,
$$

where $C$ is a finite subgroup of the center of $\tilde{G}$ and $\rho$ is a covering of topological groups. Let $P=M U$ be a parabolic subgroup of $G$. We call $\tilde{P}=\rho^{-1}(P)$ a parabolic subgroup of $\tilde{G}$. We have

$$
\tilde{P}=\tilde{M} \hat{U}
$$

where $\tilde{M}=\rho^{-1}(M)$ and $\hat{U}$ is the canonical lifting of $U$ to $\tilde{G}$ (section 2.3, [21]). Let $X(M)_{F}$ be the group of $F$-rational characters of $M$ and $\mathfrak{a}_{M}^{*}=X(M)_{F} \otimes_{\mathbb{Z}}$ $\mathbb{R}$. Given $\nu \in \mathfrak{a}_{M}^{*}$, we denote by $\exp \nu$ the corresponding unramified character of $M$. We also have an associated unramified character of $\tilde{M}$, denoted by $\widetilde{\exp } \nu$. This comes from Lemma 2.3, which gives an isomorphism between the group of unramified characters of $M$ and the group of unramified characters of $\tilde{M}$.

We define $\left(\mathfrak{a}_{M}\right)_{+}^{*}=\left\{x \in \mathfrak{a}_{M}^{*} \mid\langle x, \alpha\rangle>0, \forall \alpha \in \Pi\left(P, A_{M}\right)\right\}$. (For details, see section 2.3.) Our main result is the following theorem:

Theorem 1.1 (The Langlands quotient theorem). Let $\tilde{P}=\tilde{M} \hat{U}$ be a standard parabolic subgroup of $\tilde{G}, \nu \in\left(\mathfrak{a}_{M}\right)_{+}^{*}$ and $\tau$ the equivalence class of an irreducible tempered representation of $\tilde{M}$. Then the induced representation $i_{\tilde{G}, \tilde{M}}(\widetilde{\exp } \nu \otimes \tau)$ has a unique irreducible quotient, which we denote by $J(\tilde{P}, \nu, \tau)$. Conversely, if $\pi$ is an irreducible admissible representation of $\tilde{G}$, then there exists a unique $(\tilde{P}, \nu, \tau)$ as above such that $\pi \cong J(\tilde{P}, \nu, \tau)$.

Our approach follows a philosophy begun in [2]. The basic idea is that in light of the Bernstein-Zelevinsky geometric lemma and the Casselman criterion for temperedness, it should be possible to prove the Langlands classification using what are essentially combinatorial arguments on the exponents which occur. The exponents may be viewed as elements of $\mathfrak{a}^{*}$, where $\mathfrak{a}$ is the Lie algebra of the maximal split torus $A$ which is the Levi factor of a fixed minimal parabolic subgroup. As the positive-valued unramified characters of $A$ and those for the corresponding subgroup $\tilde{A} \subset \tilde{G}$ may be both identified with $\mathfrak{a}^{*}$, these combinatorial arguments apply to both $G$ and $\tilde{G}$. In essence, we are reproving the Langlands classification in a manner which not only covers connected reductive groups, but also their finite central extensions. We note that the case where $G=G L(n, F)$ and $\tilde{G}$ is a double cover of $G$ is discussed in [12], where the Langlands classification for $\tilde{G}$ is derived as a consequence of the Langlands classification for $G L(n, F)$.

The main technical result in this paper is the Casselman criterion for square-integrability (Theorem 3.4). The proof is based on Casselman's original work for reductive groups ([9]), using a number of structure results; some are easy to adapt, while others require subtler arguments. 
We assume $F$ has characteristic zero. If $U$ is the unipotent radical of a parabolic subgroup of $G$, then (regardless of characteristic) $U$ has a canonical lifting to $\tilde{G}$. In zero characteristic, however, this lifting is unique. This fact is used in several places (proofs of Lemma 2.7, Proposition 2.11 and Lemma 2.13).

The paper is organized as follows: In the next section, we review some structure theory for finite central extensions. In section 3, we discuss some representation theory for these groups-parabolic induction, Jacquet modules, the Casselman criterion for temperedness, etc. Finally, in section 4 we give the Langlands quotient theorem and its proof. More precisely, we present the Langlands classification in its subrepresentation form in Theorem 4.1, with the quotient form in Remark 4.2.

\section{Structure Results}

In this section, we review background material and introduce notation needed in the remainder of the paper. Let $F$ be a nonarchimedean local field of characteristic zero whose residue field has $q$ elements. We denote by $\mathcal{O}$ the ring of integers of $F$ and by $\mathfrak{p}$ the prime ideal of $F$. Let $G$ be the group of $F$-points of a connected reductive group defined over $F$.

Definition 2.1. We call $(\tilde{G}, \rho)$ a finite central extension of $G$ if the following hold:

1. $\rho: \tilde{G} \longrightarrow G$ is a surjective homomorphism of topological groups.

2. $C=k \operatorname{er}(\rho)$ is a finite subgroup of $Z(\tilde{G})$, where $Z(H)$ denotes the center of $H$.

3. $\rho$ is a topological covering (as described in [21]). In particular, there is an open neighborhood $O$ of the identity in $G$ and a homeomorphism $j: \rho^{-1}(O) \longrightarrow O \times C$ such that $p r_{1} \circ j=\rho$ on $\rho^{-1}(O)$.

We introduce some terminology for finite central extensions. If $(\tilde{G}, \rho)$ is a finite central extension of $G$, a section of $\rho$ is a continuous map $\mu: G \rightarrow \tilde{G}$ such that $\rho \circ \mu=i d_{G}$. A lifting of a subgroup $H$ of $G$ is a continuous homomorphism $s: H \rightarrow \tilde{G}$ such that $\rho \circ s=i d_{H}$. Obviously, if $G$ lifts to $\tilde{G}$ (in other words, if the sequence $1 \longrightarrow C \longrightarrow \tilde{G} \stackrel{\rho}{\longrightarrow} G \longrightarrow 1$ splits), then $\tilde{G} \cong G \times C$.

Notation Convention. Let $H$ be a subgroup of $G$. Throughout the paper, the preimage of $H$ in $\tilde{G}$ will be denoted by $\tilde{H}$ and a lifting of $H$ (if it exists) will be denoted by $\hat{H}$. Hence, $\tilde{H}=\rho^{-1}(H)$ and $\hat{H} \cong H$.

\subsection{Compact subgroups.}

Lemma 2.2. Let $(\tilde{G}, \rho)$ be a finite central extension of $G$. Then there exists a compact open subgroup of $G$ which lifts to $\tilde{G}$. 
Proof. Let $O$ be an open neighborhood of 1 as in Definition 2.1. Define $\hat{O}=j^{-1}(O \times\{1\})$. There exists an open subset $\hat{U} \subset \hat{O}$ such that $\hat{U} \hat{U} \subset \hat{O}$. Let $K$ be a compact open subgroup of $G$ such that $K \subset \rho(\hat{U})$. Denote by $\mu$ a homeomorphism $\mu: O \rightarrow \hat{O}$ such that $\rho \circ \mu=i d_{O}$. For $k_{1}, k_{2} \in K$, we have

$$
\begin{aligned}
\mu\left(k_{1} k_{2}\right) & =\mu\left(\rho\left(\mu\left(k_{1}\right)\right) \rho\left(\mu\left(k_{2}\right)\right)\right) \\
& =\mu \rho\left(\mu\left(k_{1}\right) \mu\left(k_{2}\right)\right) \\
& =\mu\left(k_{1}\right) \mu\left(k_{2}\right),
\end{aligned}
$$

so $\left.\mu\right|_{K}$ is a homomorphism.

At this point, we have the usual sort of basis of compact neighborhoods of the identity in $\tilde{G}$. In particular, let $K$ be a compact open subgroup of $G$ which lifts to $\tilde{G}$, with a lifting $s_{K}: K \rightarrow \hat{K} \subset \tilde{G}$. Let $K_{i}$ be a basis of compact open subgroups in $G$ which lie in $K$. Then $s_{K}\left(K_{i}\right)$ gives a basis of compact open subgroups in $\tilde{G}$. This makes $\tilde{G}$ an $l$-group in the terminology of $[4]$.

If $K_{\max }$ is a maximal compact open subgroup of $G$, then the preimage $\tilde{K}_{\max }=\rho^{-1}\left(K_{\max }\right)$ is a maximal compact open subgroup of $\tilde{G}$.

2.2. Unramified characters. Let us call a character of $\tilde{G}$ unramified if it is trivial on $\widetilde{{ }^{0} G}=\rho^{-1}\left({ }^{0} G\right)$, where ${ }^{0} G=\cap_{\chi}$ ker $|\chi|$, the intersection over all rational characters $\chi$ of $G$.

LEMMA 2.3. We have an isomorphism between the group $X_{u n}(G)$ of unramified characters of $G$ and the group $X_{u n}(\tilde{G})$ of unramified characters of $\tilde{G}$. It is implemented by the following (well-defined) maps:

1. If $\tilde{\chi}$ is a character of $\tilde{G}$, we define the corresponding character $\chi$ of $G$ by

$$
\chi(g)=\tilde{\chi}(\mu(g))
$$

where $\mu$ is any section of $G$ (i.e., $\mu: G \longrightarrow \tilde{G}$ with $\rho \circ \mu=i d$ ).

2. If $\chi$ is a character of $G$, we define the corresponding character $\tilde{\chi}$ of $\tilde{G}$ by

$$
\tilde{\chi}(\tilde{g})=\chi(\rho(\tilde{g})) .
$$

Proof. The observations that the maps $\chi \mapsto \tilde{\chi}$ and $\tilde{\chi} \mapsto \chi$ are welldefined, send unramified characters to unramified characters, and are inverses of each other are all straightforward calculations (or obvious). In particular, the map $\tilde{\chi} \mapsto \chi$ does not depend on the choice of a section $\mu$.

REMARK 2.4. The unramified characters (resp., positive-valued unramified characters) of $G$ correspond to elements of the dual of the real Lie algebra $\mathfrak{z}_{\mathbb{C}}^{*}$ (resp., $\mathfrak{z}^{*}$ ), where $Z$ is the center of $G$. Thus, the preceding lemma allows us to associate unramified characters (resp., positive-valued unramified characters) of $\tilde{G}$ to elements of $\mathfrak{z}_{\mathbb{C}}^{*}$ (resp., $\left.\mathfrak{z}^{*}\right)$ as well. 
2.3. Parabolic subgroups. Fix a maximal split torus $A$ in $G$. We denote by $W=W(G, A)$ the Weyl group of $G$ with respect to $A$. Let $\Phi=\Phi(G, A)$ be the set of roots. Fix a minimal parabolic subgroup $B$ containing $A$. The choice of $B$ determines the set of simple roots $\Pi$ and the set of positive roots $\Phi^{+} \subset \Phi$. If $\alpha \in \Phi^{+}$, we write $\alpha>0$.

Let $P=M U \subset G$ be a standard parabolic subgroup of $G$. We denote by $\Pi_{M} \subset \Pi$ the corresponding set of simple roots. Let $A_{M}$ be the split component of the center of $M, X(M)_{F}$ the group of $F$-rational characters of $M$. If $\Pi_{M}=\Theta$, we also use $A_{\Theta}$ to denote $A_{M}$. Hence, $A_{\emptyset}=A$ and $A_{\Pi}=A_{G}$.

The following discussion follows $[1$, Section 5$]$. The restriction homomorphism $X(M)_{F} \rightarrow X\left(A_{M}\right)_{F}$ is injective and has a finite cokernel. Therefore, we have a canonical linear isomorphism

$$
\mathfrak{a}_{M}^{*}=X(M)_{F} \otimes_{\mathbb{Z}} \mathbb{R} \stackrel{\sim}{\longrightarrow} X\left(A_{M}\right)_{F} \otimes_{\mathbb{Z}} \mathbb{R} .
$$

If $L$ is a standard Levi subgroup such that $L<M$, then

$$
A_{M} \subset A_{L} \subset L \subset M \text {. }
$$

The restriction $X(M)_{F} \rightarrow X(L)_{F}$ is injective and it induces a linear injection $\iota_{M}^{L}: \mathfrak{a}_{M}^{*} \rightarrow \mathfrak{a}_{L}^{*}$. The restriction $X\left(A_{L}\right)_{F} \rightarrow X\left(A_{M}\right)_{F}$ is surjective and it induces a linear surjection $r_{M}^{L}: \mathfrak{a}_{L}^{*} \rightarrow \mathfrak{a}_{M}^{*}$. Let $\left(\mathfrak{a}_{L}^{M}\right)^{*}$ denote the kernel of the restriction $r_{M}^{L}$. Then

$$
\mathfrak{a}_{L}^{*}=\iota_{M}^{L}\left(\mathfrak{a}_{M}^{*}\right) \oplus\left(\mathfrak{a}_{L}^{M}\right)^{*}
$$

(see $\left[1\right.$, Section 5] for details). In the case of the dual Lie algebra $\mathfrak{a}^{*}=\mathfrak{a}_{A}^{*}$ corresponding to the maximal split torus $A$ of $G$, we write simply

$$
\iota_{M}: \mathfrak{a}_{M}^{*} \rightarrow \mathfrak{a}^{*} \quad \text { and } \quad r_{M}: \mathfrak{a}^{*} \rightarrow \mathfrak{a}_{M}^{*} .
$$

Note that we have $r_{M} \circ \iota_{M}=i d$.

There is a homomorphism ([13]) $H_{M}: M \rightarrow \mathfrak{a}_{M}=\operatorname{Hom}(X(M), \mathbb{R})$ such that $q^{\left\langle\chi, H_{M}(m)\right\rangle}=|\chi(m)|$ for all $m \in M, \chi \in X(M)_{F}$. Given $\nu \in \mathfrak{a}_{M}^{*}$, let us write

$$
\exp \nu=q^{\left\langle\nu, H_{M}(\cdot)\right\rangle}
$$

for the corresponding character of $M$. As in Remark 2.4, there is then an associated unramified character of $\tilde{M}$; for clarity, we denote this character $\widetilde{\exp } \nu$.

Let $\Pi\left(P, A_{M}\right)=\left\{r_{M}(\alpha) \mid \alpha \in \Pi \backslash \Pi_{M}\right\}$ denote the set of simple roots for the pair $\left(P, A_{M}\right)$. Choose a $W$-invariant inner product $\langle\cdot, \cdot\rangle: \mathfrak{a}^{*} \times \mathfrak{a}^{*} \rightarrow \mathbb{R}$. As in [22], identifying $\mathfrak{a}_{M}^{*}$ with the subspace $\iota\left(\mathfrak{a}_{M}^{*}\right) \subset \mathfrak{a}^{*}$, we set

$$
\begin{aligned}
\left(\mathfrak{a}_{M}\right)_{+}^{*} & =\left\{x \in \mathfrak{a}_{M}^{*} \mid\langle x, \alpha\rangle>0, \forall \alpha \in \Pi\left(P, A_{M}\right)\right\}, \\
+\mathfrak{a}_{M}^{*} & =\left\{x \in \mathfrak{a}_{M}^{*} \mid x=\sum_{\alpha \in \Pi\left(P, A_{M}\right)} c_{\alpha} \alpha, c_{\alpha}>0\right\}, \\
+\overline{\mathfrak{a}}_{M}^{*} & =\left\{x \in \mathfrak{a}_{M}^{*} \mid x=\sum_{\alpha \in \Pi\left(P, A_{M}\right)} c_{\alpha} \alpha, c_{\alpha} \geq 0\right\},
\end{aligned}
$$


and $\left(\mathfrak{a}_{M}\right)_{-}^{*}=-\left(\mathfrak{a}_{M}\right)_{+}^{*}$.

Let $P=M U$ be a parabolic subgroup of $G$. We call

$$
\tilde{P}=\rho^{-1}(P)
$$

a parabolic subgroup of $\tilde{G}$. Let $\tilde{M}=\rho^{-1}(M)$ and $\hat{U}$ the canonical lifting of $U$ to $\tilde{G}$ described in the first appendix to [21]. Then

$$
\tilde{P}=\tilde{M} \hat{U}
$$

serves as the Levi factorization. Set $\tilde{A}_{M}=\rho^{-1}\left(A_{M}\right)$.

Lemma 2.5. With notation as above, $\tilde{a} \tilde{M} \tilde{a}^{-1} \subseteq \tilde{M}$, for all $\tilde{a} \in \tilde{A}_{M}$.

Proof. Let $\tilde{a} \in \tilde{A}_{M}, \tilde{m} \in \tilde{M}, a=\rho(\tilde{a}), m=\rho(\tilde{m})$. Then

$$
\rho\left(\tilde{a} \tilde{m} \tilde{a}^{-1}\right)=a m a^{-1}=m \in M,
$$

so $\tilde{a} \tilde{m} \tilde{a}^{-1} \in \rho^{-1}(M)=\tilde{M}$.

Lemma 2.6 (Bruhat decomposition). With notation as above,

$$
\tilde{G}=\coprod_{w \in W} \tilde{B} \mu(\bar{w}) \tilde{B},
$$

where $w \in W$ has representative $\bar{w} \in G$ (noting that the double-cosets are independent of the section $\mu$ and the choice of representatives $\bar{w})$. More generally, if $P=M U$ and $Q=L V$ are two standard parabolic subgroups of $G$, then

$$
\tilde{G}=\coprod_{w \in W^{M, L}} \tilde{Q} \mu(\bar{w}) \tilde{P}
$$

where $W^{M, L}=\left\{w \in W \mid w \cdot \Pi_{M} \subset \Phi^{+}, w^{-1} \cdot \Pi_{L} \subset \Phi^{+}\right\}$(see [4]).

Proof. We do the minimal parabolic case; the general case is similar.

First we check that $\tilde{G}=\cup_{w \in W} \tilde{B} \mu(\bar{w}) \tilde{B}$. For $\tilde{g} \in \tilde{G}$, write $\rho(\tilde{g})=b_{1} \bar{w} b_{2}$. Then $\tilde{g}=\mu\left(b_{1} \bar{w} b_{2}\right) c_{\tilde{g}}=\mu\left(b_{1}\right) \mu(\bar{w}) \mu\left(b_{2}\right) c$, where $c \in C$ depends on $c_{\tilde{g}}$ and the cocycle. Since $C \subset \tilde{B}$, we see that $\mu\left(b_{1}\right), \mu\left(b_{2}\right) c \in \tilde{B}$, giving the desired decomposition. To see that the double-cosets are distinct, suppose $\tilde{b}_{1} \mu(\bar{w}) \tilde{b}_{2}=$ $\tilde{b}_{1}^{\prime} \mu\left(\bar{w}^{\prime}\right) \tilde{b}_{2}^{\prime}$ with $w \neq w^{\prime}$. Applying $\rho$, we get

$$
\rho\left(\tilde{b}_{1}\right) \bar{w} \rho\left(\tilde{b}_{2}\right)=\rho\left(\tilde{b}_{1}^{\prime}\right) \bar{w}^{\prime} \rho\left(\tilde{b}_{2}^{\prime}\right) \in(B \bar{w} B) \cap\left(B \bar{w}^{\prime} B\right)=\emptyset,
$$

a contradiction. The lemma follows.

Let $P$ be the standard parabolic subgroup of $G$ corresponding to the set of simple roots $\Theta \subset \Pi$. For $\epsilon \in(0,1]$, define

$$
A_{\Theta}^{-}(\epsilon)=\left\{a \in A_{\Theta}|| \alpha(a) \mid \leq \epsilon \text {, for all } \alpha \in \Pi \backslash \Theta\right\} .
$$

We write $A_{\Theta}^{-}$for $A_{\Theta}^{-}(1)$. Define $\tilde{A}_{\Theta}^{-}=\rho^{-1}\left(A_{\Theta}^{-}\right)$. For $\alpha \in \Pi, \tilde{a} \in \tilde{A_{\emptyset}}$, we define $\alpha(\tilde{a})=(\alpha \circ \rho)(\tilde{a})$. Since $\tilde{a} \in \tilde{A}_{\Theta}^{-} \Longleftrightarrow \rho(\tilde{a}) \in A_{\Theta}^{-}$, we have

$$
\tilde{A}_{\Theta}^{-}=\left\{\tilde{a} \in \tilde{A_{\Theta}}|| \alpha(\tilde{a}) \mid \leq 1 \text {, for all } \alpha \in \Pi \backslash \Theta\right\} .
$$


If $P=M U$ is any parabolic subgroup, then $P=g^{-1} P^{\prime} g$, for some $g \in G$ and some standard parabolic subgroup $P^{\prime}$. Let $\Theta \subset \Pi$ be the set of simple roots corresponding to $P^{\prime}$. Define $A_{M}^{-}(\epsilon)=g^{-1} A_{\Theta}^{-}(\epsilon) g$ and $\tilde{A}_{M}^{-}(\epsilon)=\rho^{-1}\left(A_{M}^{-}(\epsilon)\right)$. The following lemma is analogous to [9, Proposition 1.4.3]:

Lemma 2.7. Let $\tilde{P}=\tilde{M} \hat{U}$ be a parabolic subgroup of $\tilde{G}$. If $\hat{N}_{1}, \hat{N}_{2}$ are two open compact subgroups of $\hat{U}$, then there exists $\epsilon>0$ such that $\tilde{a} \in \tilde{A}_{M}^{-}(\epsilon)$ implies $\tilde{a} \hat{N}_{2} \tilde{a}^{-1} \subseteq \hat{N}_{1}$.

Proof. Let $N_{1}=\rho\left(\hat{N}_{1}\right), N_{2}=\rho\left(\hat{N}_{2}\right)$ and let $N$ be a compact open subgroup of $U$ such that $N_{2} \subseteq N^{\ell}$, where $\ell=|C|$. According to [9, Proposition 1.4.3], there exists $\epsilon>0$ such that $a N a^{-1} \subseteq N_{1}$, for all $a \in A_{M}^{-}(\epsilon)$. Let $\tilde{a} \in \tilde{A}_{M}^{-}(\epsilon)$ and $a=\rho(\tilde{a})$. Define $s_{a}: N \rightarrow \hat{U}$ by

$$
s_{a}(x)=\tilde{a}^{-1} s_{U}\left(a x a^{-1}\right) \tilde{a},
$$

where $s_{U}$ is the unique lifting $s_{U}: U \rightarrow \hat{U}$ ([21, Appendix I]). Then $s_{a}$ is a homomorphism and $\rho \circ s_{a}=i d_{N}$. In addition, for $x \in N_{2}$ we have $s_{a}(x)=s_{U}(x)$. Therefore, $s_{a}\left(N_{2}\right)=\hat{N}_{2}$. It follows that

$$
\tilde{a} \hat{N}_{2} \tilde{a}^{-1}=s_{U}\left(a N_{2} a^{-1}\right) \subseteq s_{U}\left(N_{1}\right)=\hat{N}_{1} .
$$

Define $\tilde{A}_{\mathcal{O}}=\rho^{-1}\left(A_{\emptyset}(\mathcal{O})\right)$.

LEMMA 2.8. With notation as above,

$$
\rho^{-1}\left(A_{\Theta}^{-} \backslash A_{\emptyset}(\mathcal{O}) A_{\Pi}\right)=\tilde{A}_{\Theta}^{-} \backslash \tilde{A}_{\mathcal{O}} \tilde{A}_{\Pi} .
$$

Proof. This follows from the fact that for any two subsets $X$ and $Y$ of $G$, we have

$$
\begin{aligned}
\rho^{-1}(X Y) & =\rho^{-1}(X) \rho^{-1}(Y), \\
\rho^{-1}(X \backslash Y) & =\rho^{-1}(X) \backslash \rho^{-1}(Y) .
\end{aligned}
$$

2.4. Iwahori factorization; Cartan decomposition.

Lemma 2.9 (The Cartan decomposition). Suppose that the center of $G$ is anisotropic. Then there exists a maximal compact subgroup $\tilde{K}_{\max } \subset \tilde{G}$ such that

(a) $\tilde{G}=\tilde{K}_{\max } \tilde{P}$, for any parabolic subgroup $\tilde{P}$.

(b) $\tilde{A}_{\mathcal{O}} \subseteq \tilde{K}_{\max }$

(c) $\tilde{G}=\tilde{K}_{\max } \tilde{A}_{\emptyset}^{-} \tilde{K}_{\max }$, with the map $\tilde{a} \mapsto \tilde{K}_{\max } \tilde{a} \tilde{K}_{\max }$ establishing a bijection between $\tilde{K}_{\max } \backslash \tilde{G} / \tilde{K}_{\max }$ and $\tilde{A}_{\emptyset}^{-} / \tilde{A}_{\mathcal{O}}$. 
Proof. We apply [9, Lemma 1.4.5]. Let $K_{\max }$ be a maximal compact open subgroup of $G$ such that properties (a), (b), (c) hold for $G$. Let $\tilde{K}_{\max }=$ $\rho^{-1}\left(K_{\max }\right)$. Then (a) and (b) for $\tilde{G}$ follow directly from the corresponding properties for $G$. Also, $G=K_{\max } A_{\emptyset}^{-} K_{\max }$ implies $\tilde{G}=\tilde{K}_{\max } \tilde{A}_{\emptyset}^{-} \tilde{K}_{\max }$. It remains to prove the bijection. Suppose $\tilde{a}_{1}=\tilde{k}_{1} \tilde{a}_{2} \tilde{k}_{2}$, where $\tilde{a}_{1}, \tilde{a}_{2} \in \tilde{A}_{\emptyset}{ }^{-}$, $\tilde{k}_{1}, \tilde{k}_{2} \in \tilde{K}_{\max }$. Let $\tilde{a}=\tilde{a}_{1} \tilde{a}_{2}^{-1}$. Then $(\mathrm{c})$ for $G$ implies $\rho(\tilde{a}) \in A_{\emptyset}(\mathcal{O})$. Therefore, $\tilde{a} \in \rho^{-1}\left(A_{\emptyset}(\mathcal{O})\right)=\tilde{A}_{\mathcal{O}}$.

Proposition 2.10. Let $G$ be arbitrary. Let $\Gamma$ be a subgroup of $G$ such that $\Gamma / A_{\Pi}$ is compact, $A_{\emptyset}(\mathcal{O}) \subseteq \Gamma$ and $G=\Gamma A_{\emptyset}^{-} \Gamma$. Define $\tilde{\Gamma}=\rho^{-1}(\Gamma)$ and $\tilde{A}_{\Pi}=\rho^{-1}\left(A_{\Pi}\right)$. Then

(i) $\tilde{\Gamma} / \tilde{A}_{\Pi}$ is compact.

(ii) $\tilde{A}_{\mathcal{O}} \subseteq \tilde{\Gamma}$.

(iii) $\tilde{G}=\tilde{\Gamma} \tilde{A}_{\emptyset}^{-} \tilde{\Gamma}$.

Proof. It is clear that (ii) and (iii) hold. The map on [19, p.17], which shows $\tilde{\Gamma} / \tilde{A}_{\Pi} \cong \Gamma / A_{\Pi}$, is a homeomorphism, thus giving (i)

We define Iwahori factorizations as in [9, section 1.4]. Let $K$ be a compact open subgroup of $\tilde{G}$. We say that $K$ has an Iwahori factorization with respect to $\tilde{P}$ if the following hold:

(i) the product map is an isomorphism of $\left(\hat{U}^{-} \cap K\right) \times(\tilde{M} \cap K) \times(\hat{U} \cap K)$ with $K$

(ii) for every $\tilde{a} \in \tilde{A}_{M}^{-}, \tilde{a} \hat{U}_{K} \tilde{a}^{-1} \subseteq \hat{U}_{K}, \tilde{a}^{-1} \hat{U}_{K}^{-} \tilde{a} \subseteq \hat{U}_{K}^{-}$.

Here, $\hat{U}$ (respectively, $\hat{U}^{-}$) denotes the canonical lifting of $U$ (respectively, $U^{-}$) and $\tilde{M}=\rho^{-1}(M)$. The following proposition is analogous to $[9$, Proposition 1.4.4].

Proposition 2.11. Let $\tilde{B}$ be a minimal parabolic subgroup of $\tilde{G}$. There exists a collection $\left\{\hat{K}_{n}\right\}_{n \geq n_{0}}$, which forms a neighborhood basis of identity such that

(a) Every $\hat{K}_{n}$ is a normal subgroup of $\hat{K}_{n_{0}}$;

(b) If $\tilde{P}$ is a parabolic subgroup containing $\tilde{B}$ then $\hat{K}_{n}$ has an Iwahori factorization with respect to $\tilde{P}$;

(c) If $\tilde{P}=\tilde{M} \hat{U}$ is a parabolic subgroup containing $\tilde{B}$ then $\tilde{M}_{K_{n}}=\tilde{M} \cap \hat{K}_{n}$ has an Iwahori factorization with respect to $\tilde{M} \cap \tilde{B}$.

Proof. We have $\tilde{B}=\rho^{-1}(B)$, where $B$ is a minimal parabolic subgroup of $G$. Let $\left\{K_{n}\right\}_{n \geq 0}$ be the collection of compact subgroups of $G$ from Proposition 1.4.4 of [Ca]. Let $O$ be a compact open subgroup of $G$ which lifts to $\tilde{G}$. Fix a lifting $s_{O}: O \rightarrow \tilde{G}$. There exists $n_{1} \geq 0$ such that $K_{n} \subseteq O$, for all $n \geq n_{1}$. For $n \geq n_{1}$, define $\hat{K}_{n}=s_{O}\left(K_{n}\right)$. 
Let $U_{0}$ denote the unipotent radical of $B$. Then $U_{0}$ is conjugate to a subgroup of the upper triangular unipotent group $U(m, F) \subseteq G L(m, F)$, for some $m$ ([5, Proposition 1.10, Corollary 15.5 and Theorem 21.20]). We can assume $U_{0} \subseteq U(m, F)$ (the result in general is obtained by conjugation). For $n \geq 0$, denote by $V_{n}^{\prime}$ the kernel of the reduction $U(m, \mathcal{O}) \rightarrow U\left(m, \mathcal{O} / \mathfrak{p}^{n}\right)$. Define $V_{n}=V_{n}^{\prime} \cap U_{0}$. There exists $n_{2}$ such that $V_{n_{2}} \subseteq K_{n_{1}} \cap U_{0}$. Let $p$ be the residual characteristic of $F$ and $e$ the ramification degree. Let $C=\operatorname{ker} \rho$ and $\ell=|C|$. Write $\ell=p^{t_{0}} \ell_{0}$, where $\ell_{0}$ is relatively prime to $p$. Set $n_{3}=n_{2}+t_{0} m e$. There exists $n_{0}$ such that $K_{n_{0}} \cap U_{0} \subseteq V_{n_{3}}$. The collection $\left\{\hat{K}_{n}\right\}_{n \geq n_{0}}$ satisfies (a).

For (b), let $\hat{K}=\hat{K}_{n}$ for some $n \geq n_{0}$. Let $P=M U$ be a parabolic subgroup of $G$ containing $B$ and $\tilde{P}=\tilde{M} \hat{U}=\rho^{-1}(P)$. We claim that $\hat{K}$ has an Iwahori factorization with respect to $\tilde{P}$. Let $s_{U}, s_{U}^{-}$denote the liftings

$$
s_{U}: U \rightarrow \hat{U}, \quad s_{U}^{-}: U^{-} \rightarrow \hat{U}^{-} .
$$

We first show

$$
\left.s_{O}\right|_{K \cap U}=\left.s_{U}\right|_{K \cap U},\left.\quad s_{O}\right|_{K \cap U^{-}}=\left.s_{U}^{-}\right|_{K \cap U^{-}} .
$$

Let $u \in U$. Then there exists a unique $v \in U$ such that $u=v^{\ell}$ (see Appendix I of $[\mathrm{M}-\mathrm{W}])$. Let $\tilde{v} \in \rho^{-1}(v)$. Then $s_{U}(u)=(\tilde{v})^{\ell}$ and this does not depend on the choice of $\tilde{v}$.

If $u \in K \cap U$, then we claim that $v \in K_{n_{1}} \cap U$. Let

$$
u=1+x, \quad v=1+y,
$$

where $x_{i j} \in \mathfrak{p}^{n_{3}}$, for all $i, j=1, \ldots, m$ and $x_{i j}=0, y_{i j}=0$ for $i=$ $1, \ldots, m, j=1, \ldots, i$. Then

$$
\left(y^{k}\right)_{i j}=0, \text { for } j<i+k .
$$

We have $1+x=(1+y)^{\ell}$, so

$$
x=\ell y+\left(\begin{array}{l}
\ell \\
2
\end{array}\right) y^{2}+\cdots+\left(\begin{array}{l}
\ell \\
\ell
\end{array}\right) y^{\ell} .
$$

From (2.1) and (2.2), we obtain $y_{i, i+1} \in \mathfrak{p}^{n_{3}-t_{0} e}$, for all $i$.

It follows that

$$
\left(y^{k}\right)_{i j} \in \mathfrak{p}^{n_{3}-t_{0} e}, i=1, \ldots, m, j=1, \ldots, i+1, j \leq m .
$$

We prove by induction on $s \in\{1, \ldots, m-1\}$ that

$$
\left(y^{k}\right)_{i j} \in \mathfrak{p}^{n_{3}-t_{0} e s}, i=1, \ldots, m, j=1, \ldots, i+s, j \leq m \text {. }
$$

For $s=1$, this is (2.3). Now, assume that (2.4) is true for $s$ and prove it for $s+1$.

Let $k>1$. If $i+s+1 \leq m$, then

$$
\left(y^{k}\right)_{i, i+s+1}=\left(y \cdot y^{k-1}\right)_{i, i+s+1}=\sum_{r=1}^{m} y_{i, r}\left(y^{k-1}\right)_{r, i+s+1} .
$$


For $r=1, \ldots i$, we have $y_{i, r}=0$. For $r=i+s+1, \ldots, m$, we have $\left(y^{k-1}\right)_{r, i+s+1}=0$. Therefore,

$$
\left(y^{k}\right)_{i, i+s+1}=\sum_{r=i+1}^{i+s} y_{i, r}\left(y^{k-1}\right)_{r, i+s+1} .
$$

The inductive assumption implies $\left(y^{k}\right)_{i, i+s+1} \in \mathfrak{p}^{n_{3}-t_{0} e s}$. From (2.2), we obtain $y_{i, i+s+1} \in \mathfrak{p}^{n_{3}-t_{0} e(s+1)}$. This proves (2.4) for all $s \in\{1, \ldots, m-1\}$. It now follows that $y_{i j} \in \mathfrak{p}^{n_{2}}$, for all $i, j=1, \ldots, m$, that is, $v \in K_{n_{1}} \cap U$. Therefore

$$
s_{O}(u)=s_{O}\left(v^{\ell}\right)=\left(s_{O}(v)\right)^{\ell}=s_{U}(u),
$$

so $\left.s_{O}\right|_{K \cap U}=\left.s_{U}\right|_{K \cap U}$. Similarly, $\left.s_{O}\right|_{K \cap U^{-}}=\left.s_{U}^{-}\right|_{K \cap U^{-}}$.

We have $s_{O}(U \cap K) \subseteq s_{U}(U) \cap s_{O}(K)=\hat{U} \cap \hat{K}$. On the other hand, $\rho(\hat{U} \cap \hat{K}) \subseteq \rho(\hat{U}) \cap \rho(\hat{K})=U \cap K$, so $\hat{U} \cap \hat{K} \subseteq s_{O}(U \cap K)$. It follows that $\hat{U} \cap \hat{K}=s_{O}(U \cap K)$, i.e.,

$$
\hat{U}_{K}=s_{O}\left(U_{K}\right)=\hat{U} \cap \hat{K}
$$

In the same way we obtain

$$
\hat{U}_{K}^{-}=s_{O}\left(U_{K}^{-}\right)=\hat{U}^{-} \cap \hat{K} .
$$

Similar arguments work for $\tilde{M}_{K}$. We have

$$
\hat{M}_{K}=s_{O}\left(M_{K}\right)=\tilde{M}_{K} \cap \hat{K} .
$$

Now condition (i) for the Iwahori factorization follows immediately from (2.5), (2.6) and (2.7).

For condition (ii) for the Iwahori factorization, let $\tilde{a} \in \tilde{A}_{M}^{-}$and $a=\rho(\tilde{a})$. Then $a U_{K} a^{-1} \subset U_{K}$ and $a U_{K_{n_{1}}} a^{-1} \subset U_{K_{n_{1}}}$. Define $s_{a}: U_{K_{n_{1}}} \rightarrow \tilde{G}$ by

$$
s_{a}(x)=\tilde{a}^{-1} s_{U}\left(a x a^{-1}\right) \tilde{a} .
$$

Then $s_{a}$ is a homomorphism and $\rho \circ s_{a}=i d_{U_{K_{n_{1}}}}$. If $u \in U_{K}$, then we proved above that $u=v^{\ell}$ for $v \in U_{K_{n_{1}}}$. Then $s_{a}(u)=s_{a}\left(v^{\ell}\right)=\left(s_{a}(v)\right)^{\ell}=$ $s_{U}(u)$. It follows $\left.s_{a}\right|_{K \cap U}=\left.s_{U}\right|_{K \cap U}$ and $s_{a}\left(U_{K}\right)=\hat{U}_{K}$. Therefore, $\hat{U}_{K}=$ $\tilde{a}^{-1} s_{U}\left(a U_{K} a^{-1}\right) \tilde{a}$ and

$$
\tilde{a} \hat{U}_{K} \tilde{a}^{-1}=s_{U}\left(a U_{K} a^{-1}\right) \subseteq s_{U}\left(U_{K}\right)=\hat{U}_{K},
$$

and similarly for $U_{K}^{-}$. This proves (b). It is clear that the collection $\left\{\hat{K}_{n}\right\}_{n \geq n_{0}}$ satisfies (c). 
2.5. Central extensions of tori. Central extensions of tori are generally not commutative. An appropriate replacement for $\tilde{A}_{\emptyset}$ is the centralizer of $\tilde{M}_{\emptyset}$ in $\tilde{A}_{\emptyset}$. This fact, together with the following lemma and its proof, was communicated to us by Gordan Savin.

Lemma 2.12. Let $A$ be a $p$-adic torus. Let $n$ be a natural number. Then $A^{n}$ is an open subgroup of finite index in $A$.

Proof. Let $A_{0}$ be the maximal compact subgroup of $A$. Then $A / A_{0}$ is a lattice, hence $\left(A / A_{0}\right)^{n}$ is a full sublattice. It remains to prove that $A_{0}^{n}$ is an open compact subgroup of $A_{0}$.

Let $\mathcal{A}$ be the $p$-adic Lie algebra of $A$ and $\mathcal{A}_{0}$ a $p$-adic lattice in $\mathcal{A}$. Let $\varpi$ be the uniformizer of the $p$-adic field. Define $\mathcal{A}_{i}=\varpi^{i} \mathcal{A}_{0}$. Then there exists an integer $i_{0}$ such that the exponential map is well defined on $\mathcal{A}_{i}$ for all $i>i_{0}$. (The group $A$ is an algebraic group, hence it sits as a subgroup of $G L_{m}$, and $\mathcal{A}$ is a subalgebra of the algebra of matrices $M_{m}$. The exponential map is the usual one for matrices.) Let $A_{i}=\exp \left(\mathcal{A}_{i}\right)$. It follows that $A_{i}^{n}=A_{j}$ for every $i \geq i_{0}$, where $j-i$ is the valuation of $n$, since $n$-th power on $A_{i}$ corresponds to multiplication by $n$ on $\mathcal{A}$.

Lemma 2.13. Let $\tilde{Z}_{\emptyset}$ denote the centralizer of $\tilde{M}_{\emptyset}$ in $\tilde{A}_{\emptyset}$. Then

(a) $\tilde{Z}_{\emptyset}$ has finite index in $\tilde{A}_{\emptyset}$;

(b) $\tilde{Z}_{\Theta}=\tilde{Z}_{\emptyset} \cap \tilde{A}_{\Theta}$ is the centralizer of $\tilde{M}_{\Theta}$ in $\tilde{A}_{\emptyset}$.

Proof. (a) Let $\ell=|C|$. Then $\tilde{A}_{\emptyset}^{\ell} \subseteq \tilde{Z}_{\emptyset}$, so the statement follows from Lemma 2.12 .

(b) Let $U$ be a unipotent subgroup of $M_{\Theta}$. There exists a unique homomorphism $s: U \rightarrow \tilde{M}_{\Theta}$ such that $\rho \circ s=i d_{U}$. Set $\hat{U}=s(U)$. For $\tilde{a} \in \tilde{A}_{\Theta}$, define $s_{\tilde{a}}: U \rightarrow \tilde{M}_{\Theta}$ by $s_{\tilde{a}}(x)=\tilde{a} s(x) \tilde{a}^{-1}$. Then $\rho \circ s_{\tilde{a}}=i d_{U}$. This implies $s_{\tilde{a}}=s$, so $\tilde{a}$ commutes with any element of $\hat{U}$.

The group $M_{\Theta}$ is generated by $M_{\emptyset}$ and the root subgroups $U_{\alpha}, \alpha \in \Theta$. This implies $\tilde{M}_{\Theta}$ is generated by $\tilde{M}_{\emptyset}$ and the subgroups $\hat{U}_{\alpha}, \alpha \in \Theta$. Therefore, $\tilde{a} \in \tilde{Z}_{\Theta}=\tilde{Z}_{\emptyset} \cap \tilde{A}_{\Theta}$ commutes with any element of $\tilde{M}_{\Theta}$. This proves that $\tilde{Z}_{\Theta} \subseteq$ $C_{\tilde{A}_{\emptyset}}\left(\tilde{M}_{\Theta}\right)$. The reverse inclusion is clear since $\tilde{M}_{\emptyset} \subseteq \tilde{M}_{\Theta}$ and $\tilde{A}_{\Theta} \supseteq C_{\tilde{A}_{\emptyset}}\left(\tilde{M}_{\Theta}\right)$.

2.6. Haar measure. Let $H$ be a locally compact topological group and $\tilde{H}$ a covering group. Then $\tilde{H}$ is a locally compact topological group. Therefore, it has, up to a positive multiplicative constant, a unique left Haar measure. Let $d h$ be a left Haar measure on $H$. Suppose $H$ has an open compact subgroup $K$ which lifts to $\hat{K} \subset \tilde{H}$. Then we can choose a left Haar measure $d \tilde{h}$ on $\tilde{H}$ such that

$$
\operatorname{meas}_{\tilde{H}}(\hat{K})=\operatorname{meas}_{H}(K) .
$$


Moreover, if (2.8) holds for $K$, it holds for any compact open subgroup of $H$ which lifts to $\tilde{H}$. If (2.8) holds, we say that $d \tilde{h}$ and $d h$ are compatible.

For $\tilde{x} \in \tilde{H}$, define $\delta_{\tilde{H}}(\tilde{x})$ by

$$
\int_{\tilde{H}} f\left(\tilde{x}^{-1} \tilde{h} \tilde{x}\right) d \tilde{h}=\delta_{\tilde{H}}(\tilde{x}) \int_{\tilde{H}} f(\tilde{h}) d \tilde{h} .
$$

The definition of $\delta_{\tilde{H}}(\tilde{x})$ does not depend on the choice of $d \tilde{h}$. The function $\delta_{\tilde{H}}: \tilde{H} \rightarrow \mathbb{R}_{>0}$ is a character of $\tilde{H}$ called the modular character. The kernel of $\delta_{\tilde{H}}$ contains every compact open subgroup of $\tilde{H}$.

Proposition 2.14. Let $P=M U$ be a parabolic subgroup of $G$ and $\tilde{P}=$ $\rho^{-1}(P)$. Let $\tilde{a} \in \tilde{A}_{M}^{-}$and $a=\rho(\tilde{a})$. Then

$$
\delta_{\tilde{P}}(\tilde{a})=\delta_{P}(a) .
$$

Proof. Select compatible Haar measures on $\tilde{P}$ and $P$. Let $\hat{K} \subset \hat{O}$ be a compact open subgroup such that $\tilde{a} \hat{M}_{K} \hat{U}_{K} \tilde{a}^{-1} \subseteq \hat{M}_{K} \hat{U}_{K} \subseteq \hat{O}$, where $\hat{M}_{K}=\tilde{M} \cap \hat{K}, \hat{U}_{K}=\hat{U} \cap \hat{K}$ (cf. Lemma 2.5, Proposition 2.11). We have

$$
\text { meas }_{\tilde{P}}\left(\tilde{a} \hat{M}_{K} \hat{U}_{K} \tilde{a}^{-1}\right)=\delta_{\tilde{P}}(\tilde{a}) \operatorname{meas}_{\tilde{P}}\left(\hat{M}_{K} \hat{U}_{K}\right)
$$

and

$$
\operatorname{meas}_{\tilde{P}}\left(\tilde{a} \hat{M}_{K} \hat{U}_{K} \tilde{a}^{-1}\right)=\operatorname{meas}_{P}\left(a M_{K} U_{K} a^{-1}\right)=\delta_{P}(a) \operatorname{meas}_{P}\left(M_{K} U_{K}\right) .
$$

Since $\operatorname{meas}_{\tilde{P}}\left(\hat{M}_{K} \hat{U}_{K}\right)=\operatorname{meas}_{P}\left(M_{K} U_{K}\right)$, the claim follows.

\section{Admissible Representations}

In this section, we review some representation theoretic background in the context of finite central extensions. In particular, we discuss parabolic induction and Jacquet modules, as well as giving the Casselman criteria for square-integrability and temperedness.

An l-group is a Hausdorff topological group with a basis of neighborhoods of the identity consisting of compact open subgroups. As we observed earlier, $\tilde{G}$ is an l-group. We may then define smooth and admissible representations as usual. We give the definitions below. In addition, $\tilde{G}$ is countable at infinity (i.e., $\tilde{G}$ is a countable union of compact sets).

Let $(\pi, V)$ be a representation of $\tilde{G}$ on a complex vector space $V$. For any subgroup $K$ of $\tilde{G}$, we define $V^{K}=\{v \in V \mid \pi(k) v=v$, for all $k \in K\}$. Define $(\pi, V)$ to be a smooth representation if every $v \in V$ lies in $V^{K}$ for some compact open subgroup $K$ of $\tilde{G}$. We say that $(\pi, V)$ is admissible if it is smooth and $\operatorname{dim}\left(V^{K}\right)<\infty$ for every open subgroup $K$ of $\tilde{G}$.

Lemma 3.1 (Schur's Lemma). If $(\pi, V)$ is an irreducible smooth representation of $\tilde{G}$, then $\operatorname{End}_{\tilde{G}}(V)=\mathbb{C}$. 
Proof. Since $\tilde{G}$ is countable at infinity, we can apply Schur's Lemma from [3, section 4.2.].

As a standard consequence of Schur's Lemma, we have the following: if $(\pi, V)$ is an irreducible smooth representation of $\tilde{G}$, then there exists a character $\omega_{\pi}$ of the center $Z(\tilde{G})$ such that

$$
\pi(\tilde{z}) v=\omega_{\pi}(\tilde{z}) v \text {, for all } \tilde{z} \in Z(\tilde{G}), v \in V \text {. }
$$

We call $\omega_{\pi}$ the central character of $\pi$.

3.1. Parabolic induction and Jacquet modules. Let $\tilde{P}$ be a parabolic subgroup of $\tilde{G}$, with Levi factorization $\tilde{P}=\tilde{M} \hat{U}$ (see Section 2.3). These then satisfy the requirements of $[4,1.8]$, so we have normalized induction and Jacquet functors. More precisely, let $(\sigma, V)$ be a smooth representation of $\tilde{M}$. Then the induced representation $i_{\tilde{G}, \tilde{M}}(\sigma)$ is a representation of $\tilde{G}$ acting on the space

$$
\begin{aligned}
& i_{\tilde{G}, \tilde{M}}(V)=\{f: \tilde{G} \rightarrow V \mid f \text { is smooth and } \\
& \left.\qquad(\tilde{u} \tilde{m} \tilde{g})=\delta_{\tilde{P}}(\tilde{m})^{1 / 2} \sigma(\tilde{m}) f(\tilde{g}), \tilde{u} \in \hat{U}, \tilde{m} \in \tilde{M}, \tilde{g} \in \tilde{G}\right\}
\end{aligned}
$$

by right translation. Let $(\pi, V)$ be a smooth representation of $\tilde{G}$. Define

$$
V(\hat{U})=\operatorname{span}_{\mathbb{C}}\{\pi(\tilde{u}) v-v \mid \tilde{u} \in \hat{U}, v \in V\}
$$

and $V_{\hat{U}}=V / V(\hat{U})$. Then $r_{\tilde{M}, \tilde{G}}(\pi)$ is a representation of $\tilde{M}$ acting on $V_{\hat{U}}$ by

$$
r_{\tilde{M}, \tilde{G}}(\pi)(\tilde{m})(v+V(\hat{U}))=\delta_{\tilde{P}}(\tilde{m})^{-1 / 2} \pi(\tilde{m}) v+V(\hat{U}) .
$$

The functors $i_{\tilde{G}, \tilde{M}}$ and $r_{\tilde{M}, \tilde{G}}$ have the usual properties (see [4, Proposition 1.9]).

Lemma 3.2. Let $(\pi, V)$ be a smooth representation of $\tilde{G}$. For a compact subgroup $\hat{U}_{1}$ of $\hat{U}$, define $V\left(\hat{U}_{1}\right)=\left\{v \in V \mid \int_{\hat{U}_{1}} \pi(\hat{u}) v d \hat{u}=0\right\}$. Then

$$
V(\hat{U})=\bigcup V\left(\hat{U}_{1}\right)
$$

the union over all compact open subgroups $\hat{U}_{1}$ of $\hat{U}$.

Proof. The proof is the same as for [9, Proposition 3.2.1].

Proposition 3.3. Let $\tilde{P}=\tilde{M} \hat{U}$ and $\tilde{Q}=\tilde{L} \hat{V}$ be standard parabolic subgroups of $\tilde{G}$, where $\tilde{P}=\rho^{-1}(P), \tilde{M}=\rho^{-1}(M)$, and $\hat{U}$ is the canonical lift of $U$. If $\tau$ is an admissible representation of $\tilde{M}$, then in the Grothendieck group, we have

$$
r_{\tilde{L}, \tilde{G}} \circ i_{\tilde{G}, \tilde{M}}(\tau)=\sum_{w \in W^{M, L}} i_{\tilde{L}, \tilde{L}^{\prime}} \circ w \circ r_{\tilde{M}^{\prime}, \tilde{M}}(\tau),
$$

where $\tilde{M}^{\prime}=\tilde{M} \cap w^{-1}(\tilde{L})$ and $\tilde{L}^{\prime}=\tilde{L} \cap w(\tilde{M})$. 
Proof. This follows from Lemma 2.6 and [4, Theorem 5.2]. It is a straightforward matter to show that a subgroup $H \subset G$ which is decomposable with respect $M U$ has $\tilde{H}$ decomposable with respect to $\tilde{M} \hat{U}$, so condition (4) in Theorem 5.2 is satisfied. Conditions (1)-(3) are essentially obvious.

3.2. Square-integrable representations. Let $(\pi, V)$ be a smooth representation of $\tilde{G}$. We denote by $(\tilde{\pi}, \tilde{V})$ the contragredient of $(\pi, V)$. We have a natural pairing $\langle\rangle:, V \otimes \tilde{V} \rightarrow \mathbb{C}$ given by $\langle v, \tilde{v}\rangle=\tilde{v}(v)$. The matrix coefficient of $\pi$ associated to $v$ and $\tilde{v}$ is the function $c_{v, \tilde{v}}(\tilde{g})=\langle\pi(\tilde{g}) v, \tilde{v}\rangle$.

We define square-integrability in the usual way: an irreducible representation $\pi$ of $\tilde{G}$ is called square-integrable if it has unitary central character and $\left|c_{v, \tilde{v}}\right| \in L^{2}\left(\tilde{G} / Z_{\tilde{G}}\right)$ for all $v \in V$ and $\tilde{v} \in \tilde{V}$. An irreducible representation $\pi$ of $\tilde{G}$ is called tempered if it has unitary central character and $\left|c_{v, \tilde{v}}\right| \in$ $L^{2+\epsilon}\left(\tilde{G} / Z_{\tilde{G}}\right)$ for all $\varepsilon>0$.

Let $\tilde{P}=\tilde{M} \hat{U}$ be a standard parabolic subgroup of $\tilde{G}$. Let $\mu \in \mathfrak{a}_{M}^{*}$. We let $\exp \mu$ denote the corresponding character of $A_{M}$ (or $M$ ) and $\widetilde{\exp } \mu$ the corresponding character of $\tilde{A}_{M}$ (or $\tilde{M}$ )-cf. section 2.2 .

Let $\pi$ be an irreducible admissible representation of $\tilde{G}$. An exponent of $\pi$ with respect to $\tilde{P}$ is a $\mu \in \mathfrak{a}_{M}^{*}$ such that

$$
\widetilde{\exp } \mu \otimes \rho \leq r_{\tilde{M}, \tilde{G}}(\pi) \text { for some } \rho \text { with } \omega_{\rho} \text { unitary. }
$$

Theorem 3.4 (The Cassleman criterion for square-integrability). Suppose $\pi$ is an irreducible admissible representation of $\tilde{G}$ having unitary central character. Then $\pi$ is square-integrable if and only if for every standard parabolic subgroup $\tilde{P}=\tilde{M} \hat{U}$ and every exponent $\nu$ with respect to $\tilde{P}$, we have $\nu \in+\mathfrak{a}_{M}^{*}$.

Proof. Let $\pi$ be an irreducible admissible representation of $\tilde{G}$ having unitary central character. Observe that in section 2 we have proved the structure results which are a basis for Casselman's proof of the criterion for square-integrability [9]. More precisely, Lemma 2.7, Lemma 2.9, Proposition 2.10 and Proposition 2.11 correspond to [9, Proposition 1.4.3, Lemma 1.4.5, Proposition 1.4.6 and Proposition 1.4.4], respectively. In addition, Proposition 2.14 implies the statements corresponding to [9, Lemma 1.5 .1 and Lemma 1.5.2].

Let $v \in V, \tilde{v} \in \tilde{V}$. Let $\tilde{Z}_{\emptyset}$ denote the centralizer of $\tilde{M}_{\emptyset}$ in $\tilde{A}_{\emptyset}$. We know from Lemma 2.13 that $\tilde{Z}_{\emptyset}$ has finite index in $\tilde{A}_{\emptyset}$. Let $S$ be a finite set of representatives of $\tilde{A}_{\emptyset}^{-} /\left(\tilde{A}_{\emptyset}^{-} \cap \tilde{Z}_{\emptyset}\right)$. Let $K$ be a compact open subgroup of $\tilde{G}$, normal in $\tilde{\Gamma}(\tilde{\Gamma}$ as in Proposition 2.10), such that $v, \tilde{v}$ are fixed by $K$ and $\pi(\tilde{s}) v$ is fixed by $K$, for all $\tilde{s} \in S$. We consider square-integrability for $\left|c_{v, \tilde{v}}\right|$. More generally, we discuss integrability for $\left|c_{v, \tilde{v}}\right|^{p}, p>0$. As in the proof of $\left[9\right.$, Theorem 4.4.6], we reduce it to the question of integrability of $\left|c_{v, \tilde{v}}\right|^{p}$ on 
$K \tilde{A}_{\emptyset}^{-} K / Z(\tilde{G})$. Now

$$
\begin{aligned}
\int_{K \tilde{A}_{\bar{\emptyset}}^{-} K / Z(\tilde{G})}|\langle\pi(x) v, \tilde{v}\rangle|^{p} d x & =\sum_{\tilde{s} \in S} \int_{K\left(\tilde{A}_{\bar{\emptyset}}^{-} \cap \tilde{Z}_{\emptyset}\right) \tilde{s} K / Z(\tilde{G})}|\langle\pi(x) v, \tilde{v}\rangle|^{p} d x \\
& =\sum_{\tilde{s} \in S} \int_{K\left(\tilde{A}_{\bar{\emptyset}}^{-} \cap \tilde{Z}_{\emptyset}\right) K / Z(\tilde{G})}|\langle\pi(x) \pi(\tilde{s}) v, \tilde{v}\rangle|^{p} d x .
\end{aligned}
$$

We may replace $\pi(\tilde{s}) v$ by $v$ and consider only $\left|c_{v, \tilde{v}}\right|$ on $K\left(\tilde{A}_{\emptyset}^{-} \cap \tilde{Z}_{\emptyset}\right) K / Z(\tilde{G})$. As in [9], we reduce the problem to matrix coefficients of Jacquet modules of $\pi$. More precisely, let $\epsilon$ be as in [9, Corollary 4.3.4]. Let

$$
{ }_{\Theta} \tilde{A}_{\emptyset}^{-}(\epsilon)=\left\{\begin{array}{l|l}
\tilde{a} \in \tilde{A}_{\emptyset}^{-} \mid \begin{array}{l}
|\alpha(\tilde{a})| \leq \epsilon \\
\epsilon<|\alpha(\tilde{a})| \leq 1
\end{array} \quad \text { for } \alpha \in \Pi \backslash \Theta \in \Theta
\end{array}\right\} .
$$

Then $\tilde{A}_{\emptyset}^{-}$is the disjoint union of ${ }_{\Theta} \tilde{A}_{\emptyset}^{-}(\epsilon)$ as $\Theta$ ranges over all subsets of $\Pi$. Fix $\Theta$. Let $P=M U$ be the standard parabolic subgroup corresponding to $\Theta$. We consider integrability for $\left|c_{v, \tilde{v}}\right|^{p}$ on $K\left({ }_{\Theta} \tilde{A}_{\emptyset}^{-}(\epsilon) \cap \tilde{Z}_{\emptyset}\right) K / Z(\tilde{G})$. Using $[9$, Lemma 1.5.2] (which follows from Proposition 2.14), this reduces to $\left|c_{v, \tilde{v}}\right|^{p} \delta_{P}^{-1}$ on ${ }_{\Theta} \tilde{A}_{\emptyset}^{-}(\epsilon) \cap \tilde{Z}_{\emptyset} / Z(\tilde{G})$. Let $x, \tilde{x}$ be the images of $v, \tilde{v}$ in $V_{\hat{U}}, \tilde{V}_{\hat{U}^{-}}$. Then for $\tilde{a} \in{ }_{\Theta} \tilde{A}_{\emptyset}^{-}(\epsilon)$, we have $\langle\pi(\tilde{a}) v, \tilde{v}\rangle=\left\langle\delta_{P}^{1 / 2}(\tilde{a}) r_{M, G} \pi(\tilde{a}) x, \tilde{x}\right\rangle_{U}$ and can consider

$$
\left|\left\langle r_{M, G} \pi(\tilde{a}) x, \tilde{x}\right\rangle_{U}\right|^{p} \delta_{P}^{p / 2-1}(\tilde{a}) .
$$

Observe that Lemma 2.13 implies

$$
\tilde{A}_{\Theta} \cap \tilde{Z}_{\emptyset}=\tilde{A}_{\Theta} \cap Z(\tilde{M}) .
$$

Therefore, $\tilde{A}_{\Theta} \cap \tilde{Z}_{\emptyset}$ has generalized eigencharacters on $r_{\tilde{M}, \tilde{G}} \pi$ (central characters), and we can apply Casselman's proof. It follows that $\left|\left\langle r_{\tilde{M}}, \tilde{G} \pi(y) x, \tilde{x}\right\rangle_{U}\right|$ is square integrable on $K\left({ }_{\Theta} \tilde{A}_{\emptyset}^{-}(\epsilon) \cap \tilde{Z}_{\emptyset}\right) K / Z(\tilde{G})$ if and only if for every exponent $\nu$ with respect to $\tilde{P}$,

$$
|\widetilde{\exp } \nu(\tilde{a})|<1, \text { for all } \tilde{a} \in\left(\tilde{A}_{\Theta}^{-} \cap \tilde{Z}_{\emptyset}\right) \backslash \tilde{A}_{\mathcal{O}} \tilde{A}_{\Pi} .
$$

For any $\tilde{a} \in \tilde{A}_{\Theta}$ we have $\tilde{a}^{\ell} \in Z(\tilde{M})$, where $\ell=|C|$. Condition (3.2) is then equivalent to $|\widetilde{\exp } \nu(\tilde{a})|<1$ for all $\tilde{a} \in \tilde{A}_{\Theta}^{-} \backslash \tilde{A}_{\mathcal{O}} \tilde{A}_{\Pi}$. In summary, $\pi$ is squareintegrable if and only if for every standard parabolic subgroup $\tilde{P}=\tilde{M} \hat{U}$ and every exponent $\nu$ with respect to $\tilde{P}$, we have $|\widetilde{\exp } \nu(\tilde{a})|<1$ for all $\tilde{a} \in$ $\tilde{A}_{\Theta}^{-} \backslash \tilde{A}_{\mathcal{O}} \tilde{A}_{\Pi}$. According to Lemma 2.3 and Lemma 2.8, this is equivalent to $|\exp \nu(a)|<1$ for all $a \in A_{\Theta}^{-} \backslash A_{\emptyset}(\mathcal{O}) A_{\Pi}$, i.e., $\nu \in+\mathfrak{a}_{M}^{*}$.

Proposition 3.5 (The Casselman criterion for temperedness). Suppose $\pi$ is an irreducible admissible representation of $\tilde{G}$ having unitary central character. Then $\pi$ is tempered if and only if for every standard parabolic subgroup $\tilde{P}=\tilde{M} \hat{U}$ and every exponent $\nu$ with respect to $\tilde{P}$, we have $\nu \in+\overline{\mathfrak{a}}_{M}^{*}$. 
Proof. We apply the proof of the previous theorem for $p=2+\epsilon, \epsilon>0$. Define $\Psi(\tilde{a})=\left\langle\left(r_{M, G} \pi\right)(\tilde{a}) u, \tilde{u}\right\rangle_{U}$. Then (3.1) is equal to

$$
|\Psi|^{p} \delta_{P}^{p / 2-1}=|\Psi|^{2+\epsilon} \delta_{P}^{\epsilon / 2}=\left|\Psi \delta_{P}^{\epsilon /(2(2+\epsilon))}\right|^{2+\epsilon} .
$$

So, for temperedness, we need for every central character $\chi$ of $\Psi, \tilde{a} \in \tilde{A}_{\Theta}^{-} \backslash$ $\tilde{A}_{\mathcal{O}} \tilde{A}_{\Pi}$,

$$
|\chi(\tilde{a})|<\delta_{P}^{-\epsilon /(2(2+\epsilon))}(\tilde{a}), \quad \text { for all } \epsilon>0 .
$$

We have $\delta_{P}(\tilde{a})<1$, for $\tilde{a} \in \tilde{A}_{\Theta}^{-} \backslash \tilde{A}_{\mathcal{O}} \tilde{A}_{\Pi}$. Therefore, the right hand side of the above inequality is greater than 1 for all $\epsilon>0$. Since $\lim _{\epsilon \rightarrow 0} \epsilon /(2(2+\epsilon))=0$, we obtain $|\chi(\tilde{a})| \leq 1$.

REMARK 3.6. To faciliate the combinatorial arguments in section 4 , we take a moment to reformulate this as in [2]. If $\pi$ is an irreducible admissible representation of $\tilde{G}$, let

$\mathcal{M}_{\text {min }}(\pi)=\left\{\tilde{L}\right.$ standard Levi $\mid r_{\tilde{L}, \tilde{G}}(\pi) \neq 0$ but $r_{\tilde{H}, \tilde{G}}(\pi)=0$ for all $\left.\tilde{H}<\tilde{L}\right\}$.

Now, set

$$
\begin{aligned}
& \operatorname{Exp}(\pi)=\left\{\iota(\mu) \mid \widetilde{\exp } \mu \otimes \rho \leq r_{\tilde{L}, \tilde{G}}(\pi) \text { for some } \rho\right. \\
& \text { with } \left.\omega_{\rho} \text { unitary and } \tilde{L} \in \mathcal{M}_{\min }(\pi)\right\} .
\end{aligned}
$$

It now follows from [2, Lemma 4.3] that if $\pi$ is an irreducible unitary representation, then $\pi$ is tempered if and only if $\nu \in+\overline{\mathfrak{a}}^{*}$ for every $\nu \in \operatorname{Exp}(\pi)$.

\section{The LANGLANDS CLASSIFICATION}

In this section, we state and prove the Langlands classification for finite central extensions. The subrepresentation version is Theorem 4.1; the quotient version Theorem 1.1 and Remark 4.2. The proof is done in the subrepresentation setting for technical reasons: if $\pi \cong L(\tilde{P}, \nu, \tau)$, then $\widetilde{\exp } \nu \otimes \tau \leq r_{\tilde{M}, \tilde{G}}(\pi)$.

A set of Langlands data for $\tilde{G}$ is a triple $(\tilde{P}, \nu, \tau)$ with the following properties:

(1) $\tilde{P}=\tilde{M} \hat{U}$ is a standard parabolic subgroup of $\tilde{G}$,

(2) $\nu \in\left(\mathfrak{a}_{\tilde{M}}\right)_{-}^{*}$, and

(3) $\tau$ is (the equivalence class of) an irreducible tempered representation of $\tilde{M}$.

We now state the Langlands classification in the subrepresentation setting.

TheOrem 4.1 (The Langlands classification). Suppose $(\tilde{P}, \nu, \tau)$ is a set of Langlands data for $\tilde{G}$. Then the induced representation $i_{\tilde{G}, \tilde{M}}(\widetilde{\exp } \nu \otimes \tau)$ has a unique irreducible subrepresentation, which we denote by $L(\tilde{P}, \nu, \tau)$. 
Conversely, if $\pi$ is an irreducible admissible representation of $\tilde{G}$, then there exists a unique $(\tilde{P}, \nu, \tau)$ as above such that $\pi \cong L(\tilde{P}, \nu, \tau)$.

The proof of this result has three main parts. First, we show that $L(\tilde{P}, \nu, \tau)$ is well-defined-i.e., that $i_{\tilde{G}, \tilde{M}}(\widetilde{\exp } \nu \otimes \tau)$ has a unique irreducible subrepresentation, see Corollary 4.4. Then, in Proposition 4.8, we show that any irreducible admissible $\pi$ may be written in the form $L(\tilde{P}, \nu, \tau)$ (existence of Langlands data). Finally, in Proposition 4.10, we show that if $\left(\tilde{P}_{1}, \nu_{1}, \tau_{1}\right)$ and $\left(\tilde{P}_{2}, \nu_{2}, \tau_{2}\right)$ are two such triples and $L\left(\tilde{P}_{1}, \nu_{1}, \tau_{1}\right) \cong L\left(\tilde{P}_{2}, \nu_{2}, \tau_{2}\right)$, then $\tilde{P}_{1}=\tilde{P}_{2}, \nu_{1}=\nu_{2}$, and $\tau_{1} \cong \tau_{2}$ (uniqueness of Langlands data).

REMARK 4.2. This theorem describes the Langlands classification in the subrepresentation setting. It can also be formulated in the quotient setting (see Theorem 1.1), in which case one has $\nu \in\left(\mathfrak{a}_{\tilde{M}}\right)_{+}^{*}$ (and the associated irreducible representation appears as a quotient).

To see this, let $\pi$ be an irreducible admissible representation. Let $(\tilde{P}, \nu, \tau)$ be the Langlands data for $\tilde{\pi}$, the contragredient of $\pi$. Since $\tilde{\pi}$ is the unique irreducible subrepresentation of $i_{\tilde{G}, \tilde{M}}(\widehat{\exp } \nu \otimes \tau)$, we see $\pi$ is the unique irreducible quotient of

$$
i_{\tilde{G}, \tilde{M}}(\widetilde{\exp } \nu \otimes \tau)^{\sim} \cong i_{\tilde{G}, \tilde{M}}(\widetilde{\exp }(-\nu) \otimes \tilde{\tau}) .
$$

We have $-\nu \in\left(\mathfrak{a}_{\tilde{M}}\right)_{+}^{*}$ and $\tilde{\tau}$ tempered, as needed. (The quotient and subrepresentation data for a given $\pi$ should be related as in [14, Lemma 1.1]. However, the argument there relies on the characterization of the Langlands quotient in terms of standard intertwining operators, which we do not have at this point.)

As in $\left[6\right.$, Chapter XI], set $\mathcal{F}=\sum \mathbb{R} \alpha_{i}$, where the sum is over the simple roots $\Pi=\left\{\alpha_{1}, \ldots, \alpha_{n}\right\}$. Then $\mathfrak{a}^{*}=\mathfrak{z}^{*} \oplus \mathcal{F}$, where $\mathfrak{z}^{*}=\left\{x \in \mathfrak{a}^{*} \mid\langle x, \alpha\rangle=\right.$ 0 , for all $\alpha \in \Pi\}$. For $\nu \in \mathfrak{a}^{*}$, we define $\nu_{0}$ to be the point in $\overline{\left(\mathfrak{a}_{-}^{*}\right)} \cap \mathcal{F}$ which is closest to $\nu$. Define $\beta_{1}, \ldots, \beta_{n} \in \mathcal{F}$ by $\left\langle\beta_{i}, \alpha_{j}\right\rangle=\delta_{i j}$. Then $\mathcal{F}=\sum \mathbb{R} \beta_{i}$. More generally, if $I \subset\{1, \ldots, n\}$, then $\mathfrak{a}^{*}=\mathfrak{z}^{*}+\sum_{i \notin I} \mathbb{R} \beta_{i}+\sum_{i \in I} \mathbb{R} \alpha_{i}$ (see [6, Chapter IV.6.6]). Note that if $M$ is the standard Levi factor with $\Pi_{M}=$ $\left\{\alpha_{i} \mid i \in I\right\}$, then $i_{M}\left(\mathfrak{a}_{M}^{*}\right)=\mathfrak{z}^{*}+\sum_{i \notin I} \mathbb{R} \beta_{i}$. The set of simple roots $\Pi$ is a basis of an abstract root system in $\mathcal{F}$. Note that if $\nu=z+\sum_{i \notin F} a_{i} \beta_{i}+\sum_{i \in F} a_{i} \alpha_{i}$ with $a_{i}<0$ for all $i \notin F$ and $a_{i} \geq 0$ for all $i \in F$, then

$$
\nu_{0}=\sum_{i \notin F} a_{i} \beta_{i}
$$

([16, Lemma 8.56]).

We now recall the following result ([2, Lemma 3.3]). 
Lemma 4.3. Let $\tilde{P}=\tilde{M} \hat{U}$ be a standard parabolic subgroup of $\tilde{G}$. Let $F \subset\{1, \ldots, n\}$ be such that $\Pi_{\tilde{M}}=\left\{\alpha_{i} \mid i \in F\right\}$. If

$$
\begin{aligned}
& x \in T_{F}=\left\{x \in \mathcal{F} \mid x=\sum_{i \notin F} c_{i} \beta_{i}+\sum_{j \in F} c_{j} \alpha_{j}\right. \\
& \text { with } \left.c_{i}<0 \text { for } i \notin F \text { and } c_{j} \geq 0 \text { for } j \in F\right\}
\end{aligned}
$$

and $w \in W^{M, A}$ (cf. Lemma 2.6) with $w \neq 1$, then $(w x)_{0} \neq x_{0}$.

Corollary 4.4. Let $(\tilde{P}, \nu, \tau)$ be a set of Langlands data for $\tilde{G}$. Then $i_{\tilde{G}, \tilde{M}}(\widetilde{\exp } \nu \otimes \tau)$ has a unique irreducible subrepresentation (denoted $L(\tilde{P}, \nu, \tau)$ above).

Proof. We use the following standard result ([9, Proposition 2.1.9], [11, Lemma 8.2], [23, section I.3] for $G$; it is essentially the same for $\tilde{G})$ : If $(\rho, V)$ is an admissible representation of $\tilde{M}$ and $\omega$ is a character of $Z_{\tilde{M}}$, write

$V_{\omega}=\left\{v \in V \mid\right.$ there is an $n \in \mathbb{N}$ such that $[\rho(z)-\omega(z)]^{n} v=0$ for all $\left.z \in Z_{\tilde{M}}\right\}$. Then $V=\oplus_{\omega} V_{\omega}$ as a direct sum of $\tilde{M}$-modules. In particular, let $\rho=r_{\tilde{M}, \tilde{G}}(\pi)$ and $\lambda=\widetilde{\exp } \nu \otimes \omega_{\tau}$. By Lemma 4.3 and Proposition 3.3, $V_{\lambda}$ is just the $\tilde{M}$ module $\widetilde{\exp } \nu \otimes \tau$ (as it is the unique subquotient of $r_{\tilde{M}, \tilde{G}}(\pi)$ having this central character), so appears as a direct summand in $r_{\tilde{M}, \tilde{G}}(\pi)$. The corollary now follows from Frobenius reciprocity.

REMARK 4.5. This actually shows more: it also follows that $L(\tilde{P}, \nu, \tau)$ appears with multiplicity one in $i_{\tilde{G}, \tilde{M}}(\widetilde{\exp } \nu \otimes \tau)$. Further, $\widetilde{\exp } \nu \otimes \tau$ is the unique irreducible subquotient of $r_{\tilde{M}, \tilde{G}} \circ i_{\tilde{G}, \tilde{M}}(\widetilde{\exp } \nu \otimes \tau)$ having its central character.

The proof of existence is based in part on that given in [16], which borrows from the original proof in [20].

Definition 4.6. For $\nu, \nu^{\prime} \in \mathfrak{a}^{*}$, we write $\nu \preceq \nu^{\prime}$ if $\left\langle\nu^{\prime}-\nu, \beta_{i}\right\rangle \geq 0$ for all $i$.

We note that $\preceq$ defines a partial order on $\mathfrak{a}^{*}$. We now have the following standard lemma:

LEMMA 4.7. If $\nu \preceq \nu^{\prime}$, then $\nu_{0} \preceq \nu_{0}^{\prime}$.

Proof. This is [16, Lemma 8.59].

For $F \subset\{1, \ldots, n\}$, let $T_{F}$ be defined as in Lemma 4.3. The sets $T_{F}$ partition $\mathcal{F}$ into $2^{n}$ disjoint subsets (this follows immediately from $[6$, Lemmas IV.6.9-IV.6.11]). For $\nu \in \mathfrak{a}^{*}$, we define $F(\nu) \subset\{1, \ldots, n\}$ to be the unique subset for which we have $\nu \in \mathfrak{z}^{*}+T_{F(\nu)}$. 
Proposition 4.8. Let $\pi$ be an irreducible admissible representation. Then there exists a triple $(\tilde{P}, \nu, \tau)$ satisfying the requirements for Langlands data and such that $\pi \hookrightarrow \operatorname{Ind} d_{\tilde{P}}^{\tilde{G}}(\widetilde{\exp } \nu \otimes \tau)$.

Proof. Choose $\mu \in \operatorname{Exp}(\pi)$ such that $\mu_{0}$ is minimal with respect to $\preceq$ $(\operatorname{Exp}(\pi)$ as in Remark 3.6). Write

$$
\mu=z+\sum_{i \notin F(\mu)} a_{i} \beta_{i}+\sum_{j \in F(\mu)} a_{j} \alpha_{j}
$$

with $a_{i}<0$ for $i \notin F(\mu)$ and $a_{j} \geq 0$ for $j \in F(\mu)$. Let $\tilde{P}=\tilde{P}_{F(\mu)}=\tilde{M} \hat{U}$. Set

$$
\nu=r_{M}\left(\sum_{i \notin F(\mu)} a_{i} \beta_{i}\right),
$$

(notation as in Section 2.3). We have $\nu \in\left(\mathfrak{a}_{M}^{*}\right)_{-}$.

By definition, $\mu \in \operatorname{Exp}(\pi)$ means $\mu=\iota\left(\mu^{\prime}\right)$ for some $\widetilde{\exp } \mu^{\prime} \otimes \rho \leq r_{\tilde{L}, \tilde{G}}(\pi)$, with $\rho$ an irreducible unitary supercuspidal representation $\rho$ and $\mu^{\prime} \in \mathfrak{a}_{L}^{*}$. We now claim there is some $\widetilde{\exp } \nu \otimes \theta \in r_{\tilde{M}, \tilde{G}}(\pi)$ such that $\widetilde{\exp } \mu^{\prime} \otimes \rho \leq$ $r_{\tilde{L}, \tilde{M}}(\widetilde{\exp } \nu \otimes \theta)$. If we show $\tilde{L} \leq \tilde{M}$, this follows immediately from taking Jacquet modules in stages. To show $\tilde{L} \leq \tilde{M}$, we argue that if $i \notin F(\mu)$ (i.e., $\alpha_{i} \notin \Pi_{\tilde{M}}$ ), then $\alpha_{i} \notin \Pi_{\tilde{L}}$. To this end, note that if $\alpha_{i} \in \Pi_{\tilde{L}}$, then $\left\langle\iota\left(\mu^{\prime}\right), \alpha_{i}\right\rangle=0$. On the other hand, if $i \notin F(\mu)$, then

$$
\left\langle\mu, \alpha_{i}\right\rangle=c_{i}+\sum_{j \in F(\mu)} c_{j}\left\langle\alpha_{j}, \alpha_{i}\right\rangle<0
$$

since $\left\langle\alpha_{j}, \alpha_{i}\right\rangle \leq 0$ for $j \neq i$. The claim follows. Note that any $\lambda \in \operatorname{Exp}(\widetilde{\exp } \nu \otimes$ $\theta)$ may be written

$$
\lambda=z+\sum_{i \notin F(\mu)} a_{i} \beta_{i}+\sum_{j \in F(\mu)} b_{j} \alpha_{j}
$$

(not necessarily having $b_{j} \geq 0$ ).

It remains to check that $\theta$ is tempered for any such $\theta$. By the Casselman criterion, this requires showing $b_{j} \geq 0$ for all $j \in F(\mu)$. Suppose this were not the case. Let $F^{\prime}=\left\{j \in F(\mu) \mid b_{j}<0\right\}$. Then,

$$
\begin{aligned}
\lambda & =z+\sum_{i \notin F(\mu)} a_{i} \beta_{i}-\sum_{j \in F^{\prime}}\left(-b_{j}\right) \alpha_{j}+\sum_{j \in F(\mu)-F^{\prime}} b_{j} \alpha_{j} \\
& \preceq z+\sum_{i \notin F(\mu)} a_{i} \beta_{i}+\sum_{j \in F(\mu)-F^{\prime}} b_{j} \alpha_{j}=\lambda^{\prime} .
\end{aligned}
$$


Since $\mu_{0}=\sum_{i \notin F(\mu)} a_{i} \beta_{i}=\lambda_{0}^{\prime}$, it follows from Lemma 4.7 that

$$
\begin{gathered}
\lambda \preceq \lambda^{\prime} \\
\Downarrow \\
\lambda_{0} \preceq \lambda_{0}^{\prime}=\mu_{0} .
\end{gathered}
$$

By the minimality of $\mu_{0}$, we see that $\lambda_{0}=\mu_{0}$. Therefore, $F(\lambda)=F(\mu)$, so $F^{\prime}=\emptyset$. Thus $b_{i} \geq 0$ for all $i \in F(\mu)$, implying temperedness.

That $\pi \hookrightarrow \operatorname{Ind}_{\tilde{P}}^{\tilde{G}}(\widetilde{\exp } \nu \otimes \tau)$ for some irreducible tempered $\tau$ now follows immediately from Frobenius reciprocity and central character considerations as in Corollary 4.4 .

The proof of uniqueness is based on that in [10]. Let $\gamma=\sum_{i=1}^{n} \beta_{i}$. Note that $\left\langle\sum a_{i} \alpha_{i}, \gamma\right\rangle=\sum a_{i}$.

Lemma 4.9. Suppose

$$
\nu=\sum_{i \notin F} c_{i} \beta_{i}+\sum_{j \in F} c_{j} \alpha_{j},
$$

with $c_{i}<0$ for $i \notin F$ and $c_{j} \geq 0$ for $j \in F$. Let $\tilde{M}=\tilde{M}_{F}$ and $w \in W^{M, A}$ with $w \neq 1$. Then $\langle w \nu, \gamma\rangle>\langle\nu, \gamma\rangle$.

Proof. Observe that

$$
\langle w \nu, \gamma\rangle=\sum_{i \notin F} c_{i}\left\langle w \beta_{i}, \gamma\right\rangle+\sum_{j \in F} c_{j}\left\langle w \alpha_{j}, \gamma\right\rangle
$$

Since $w \in W^{M, A}$, we have $w \alpha_{j}>0$ for all $j \in F$. It follows immediately that

$$
\left\langle w \alpha_{j}, \gamma\right\rangle \geq\left\langle\alpha_{j}, \gamma\right\rangle
$$

for all $j \in F$. On the other hand, since $\gamma \in \mathfrak{a}_{+}^{*}$, it follows from the Corollary to $[7$, Proposition 18 , chapter 6 , section 1$]$ that

$$
\left\langle w \beta_{k}, \gamma\right\rangle \leq\left\langle\beta_{k}, \gamma\right\rangle
$$

for all $k$ with strict inequality for at least one $k$. The lemma now follows.

Proposition 4.10. Suppose $\left(\tilde{P}_{1}, \nu_{1}, \tau_{1}\right)$ and $\left(\tilde{P}_{2}, \nu_{2}, \tau_{2}\right)$ are Langlands data such that $L\left(\tilde{P}_{1}, \nu_{1}, \tau_{1}\right) \cong L\left(\tilde{P}_{2}, \nu_{2}, \tau_{2}\right)$. Then, $\tilde{P}_{1}=\tilde{P}_{2}, \nu_{1}=\nu_{2}$, and $\tau_{1} \cong \tau_{2}$.

Proof. Write $\pi=L\left(\tilde{P}_{1}, \nu_{1}, \tau_{1}\right) \cong L\left(\tilde{P}_{2}, \nu_{2}, \tau_{2}\right)$. For $i=1,2$, let $\mu_{i} \in$ $\operatorname{Exp}\left(\widetilde{\exp } \nu_{i} \otimes \tau_{i}\right)$ with $\left\langle\mu_{i}, \gamma\right\rangle$ minimal. Since $\pi \hookrightarrow \operatorname{Ind}_{\tilde{P}_{2}}^{\tilde{G}}\left(\widetilde{\exp } \nu_{2} \otimes \tau_{2}\right)$, it follows from Frobenius reciprocity and the Bernstein-Zelevinsky/Casselman result (Proposition 3.3) that $\mu_{2}=w \mu_{1}^{\prime}$ for some $w \in W^{M_{1}, A}$ and $\mu_{1}^{\prime} \in \operatorname{Exp}\left(\widetilde{\exp } \nu_{1} \otimes\right.$ $\left.\tau_{1}\right)$. By the preceding lemma,

$$
\left\langle\mu_{2}, \gamma\right\rangle \geq\left\langle\mu_{1}^{\prime}, \gamma\right\rangle \geq\left\langle\mu_{1}, \gamma\right\rangle
$$


with equality possible only if $w=1$, i.e., $\mu_{2}=\mu_{1}^{\prime}$. Similarly, $\left\langle\mu_{1}, \gamma\right\rangle \geq\left\langle\mu_{2}, \gamma\right\rangle$. In particular, we must have equality, so $\mu_{2}=\mu_{1}^{\prime}$. Now, any exponent in $\operatorname{Exp}\left(\widetilde{\exp } \nu_{1} \otimes \tau_{1}\right)$ has the form $z+\sum_{i \notin F_{1}} c_{i} \beta_{i}+\sum_{j \in F_{1}} c_{j} \alpha_{j}$ with $\iota\left(\nu_{1}\right)=$ $z+\sum_{i \notin F_{1}} c_{i} \beta_{i}$ (so $\nu_{1} \in\left(\mathfrak{a}_{M}^{*}\right)_{-} \Rightarrow c_{i}<0$ for $i \notin F_{1}$ ) and $\sum_{j \in F_{1}} c_{j} \alpha_{j}$ an exponent for the tempered representation $\tau_{1}$ (so $c_{j} \geq 0$ for all $j \in F_{1}$ by the Casselman criterion). In particular, all the exponents in $\operatorname{Exp}\left(\widetilde{\exp } \nu_{1} \otimes \tau_{1}\right)$ belong to $\mathfrak{z}^{*}+T_{F_{1}}$, so $\mu_{2} \in \mathfrak{z}^{*}+T_{F_{1}}$, i.e., $F_{1}=F_{2}$. Thus, $\tilde{P}_{1}=\tilde{P}_{2}$; write $\tilde{P}=\tilde{M} \hat{U}$ for this parabolic subgroup. Then, we also have

$$
\nu_{1}=r_{M}\left(\mu_{1}^{\prime}\right)=r_{M}\left(\mu_{2}\right)=\nu_{2} .
$$

That $\tau_{1} \cong \tau_{2}$ now follows as in [2, Proposition 5.3/Corollary 5.4] (noting that the key ingredient in that proof (see [2, Lemma 3.3], which is Lemma 4.3 above) is combinatorial in nature and can be applied to the case of central extensions).

REMARK 4.11. Based on the real case and the $p$-adic version in BorelWallach, one might hope to have $\nu$ minimal with respect to $\succeq$ in the standard module. This would require a nontrivial refinement of [2, Lemma 3.3], but would also be enough to show the uniqueness above.

\section{ACKNOWLEDGEMENTS.}

We would like to thank Goran Muić, who suggested this project. We would also like to thank Bill Casselman, David Vogan, and the referees for their valuable comments. D.B. thanks Werner Müller and Mathematical Institute of the University of Bonn for their hospitality during her three month research stay, where a part of this work was done. In an earlier version of this paper, we had an assumption that $G$ is split; we are grateful to Gordan Savin for his advice on how to remove the assumption.

\section{REFERENCES}

[1] J. Arthur, An introduction to the trace formula, in Harmonic analysis, the trace formula, and Shimura varieties, Clay Math. Proc. 4, Amer. Math. Soc., Providence, RI, 2005, 1-263.

[2] D. Ban and C. Jantzen, Jacquet modules and the Langlands classification, Michigan Math. J. 56 (2008), 637-653.

[3] J. Bernstein, Representations of $p$-adic groups, Lectures, Harvard University, Fall 1992.

[4] I. Bernstein and A. Zelevinsky, Induced representations of reductive p-adic groups I, Ann. Sci. École Norm. Sup. 10 (1977), 441-472.

[5] A. Borel, Liner Algebraic Groups, 2nd Edition, Springer-Verlag, New York, 1991.

[6] A. Borel and N. Wallach, Continuous cohomology, discrete subgroups, and representations of reductive groups, Princeton University Press, Princeton, 1980.

[7] N. Bourbaki, Lie Groups and Lie Algebras (Chapters 4-6), Springer-Verlag, Berlin, 2002.

[8] J.-L. Brylinski and P. Deligne, Central extensions of reductive groups by K2, Publ. Math. Inst. Hautes Études Sci. 94 (2001), 5-85. 
[9] W. Casselman, Introduction to the theory of admissible representations of p-adic reductive groups, preprint.

[10] S. Evens, The Langlands classification for graded Hecke algebras, Proc. Amer. Math. Soc. 124 (1996), 1285-1290.

[11] R. Gustafson, The degenerate principal series for $S p(2 n)$, Mem. Amer. Math. Soc. 33 (1981).

[12] M. Hanzer and G. Muić, Parabolic induction and Jacquet functors for mateplectic groups, J. Algebra 323 (2010), 241-260.

[13] Harish-Chandra, Harmonic analysis on reductive p-adic groups, Proceedings of Symposia in Pure Mathematics 26 (1973), 167-192.

[14] C. Jantzen, Some remarks on degenerate principal series, Pacific J. Math. 186 (1998), 67-87.

[15] D. Kazhdan and S. Kazhdan, Metaplectic forms, Inst. Hautes Études Sci. Publ. Math. 59 (1984), 35-142.

[16] A. Knapp, Representation Theory of Semisimple Groups; An Overview Based on Examples, Princeton University Press, Princeton, 1986.

[17] T. Konno, A note on the Langlands classification and irreducibility of induced representations of p-adic groups, Kyushu J. Math. 57 (2003), 383-409.

[18] S. Kudla, On the local theta-correspondence, Invent. Math. 83 (1986), 229-255.

[19] S. Lang, Algebra, 3rd revised ed., New York, Springer, 2002.

[20] R. Langlands, On the classification of irreducible representations of real algebraic groups, in Representation theory and harmonic analysis on semisimple Lie groups, Amer. Math. Soc., Providence, 1989, 101-170.

[21] C. Mœglin and J.-L. Waldspurger, Spectral Decomposition and Eisenstein Series, Cambridge University Press, Cambridge, 1995.

[22] A. Silberger, The Langlands quotient theorem for p-adic groups, Math. Ann. 236 (1978), 95-104.

[23] J.-L. Waldspurger, La formule de Plancherel pour les groupes p-adiques (d'après Harish-Chandra), J. Inst. Math. Jussieu 2 (2003), 235-333.

[24] M. Weissman, Metaplectic tori over local fields, Pacific J. Math. 241 (2009), 169-200.

D. Ban

Department of Mathematics

Southern Illinois University

Carbondale, IL 62901

USA

E-mail: dban@math.siu.edu

C. Jantzen

Department of Mathematics

East Carolina University

Greenville, NC 27858

USA

E-mail: jantzenc@ecu.edu

Received: 3.9.2012.

Revised: 25.3.2013. 